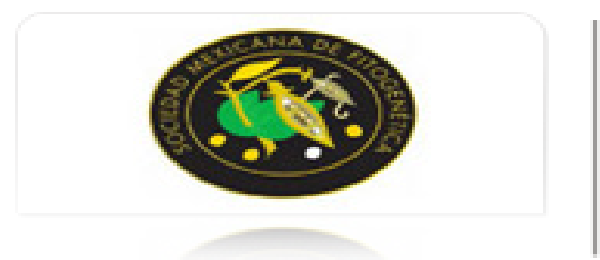

Revista Fitotecnia Mexicana

ISSN: 0187-7380

revfitotecniamex@gmail.com

Sociedad Mexicana de Fitogenética, A.C.

México

Martínez-Gutiérrez, Gabino A.; Ortiz-Hernández, Yolanda D.; López-Pozos, Roberto OXIGENACIÓN DE LA SOLUCIÓN NUTRITIVA RECIRCULANTE Y SU EFECTO EN TOMATE Y LECHUGA

Revista Fitotecnia Mexicana, vol. 35, núm. 5, septiembre, 2012, pp. 49-52 Sociedad Mexicana de Fitogenética, A.C.

Chapingo, México

Disponible en: http://www.redalyc.org/articulo.oa?id=61024388009

Cómo citar el artículo

- Número completo

- Más información del artículo

Página de la revista en redalyc.org

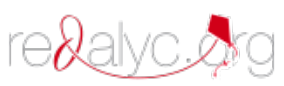

Sistema de Información Científica

Red de Revistas Científicas de América Latina, el Caribe, España y Portugal Proyecto académico sin fines de lucro, desarrollado bajo la iniciativa de acceso abierto 


\section{OXIGENACIÓN DE LA SOLUCIÓN NUTRITIVA RECIRCULANTE Y SU EFECTO EN TOMATE Y LECHUGA}

\section{OXYGENATION OF THE RECIRCULATING NUTRIENT SOLUTION AND ITS EFFECT ON TOMATO AND LETTUCE}

\author{
Gabino A. Martínez-Gutiérrez ${ }^{1 *}$, Yolanda D. \\ Ortiz-Hernández ${ }^{1}$ y Roberto López-Pozos ${ }^{2}$
}

${ }^{1}$ Centro Interdisciplinario de Investigación para el Desarrollo Rural Integral Unidad Oaxaca, Instituto Politécnico Nacional. Hornos 1003. Indeco. Xoxocotlan, Oaxaca. Oax. Tel. y Fax 01 (951) 5170610 Ext. 82761²Universidad del Mar, Campus Puerto Escondido, Oaxaca.

${ }^{*}$ Autor para correspondencia (gamartinezg@ipn.mx)

\section{RESUMEN}

La oxigenación inadecuada de la solución nutritiva afecta la absorción de nutrientes y de agua, con efectos negativos en el desarrollo de los cultivos. En este estudio se evaluó la incorporación de oxígeno en un sistema hidropónico recirculante por dos métodos pasivos: aumentar la pendiente del contenedor y agregar saltos hidráulicos en cultivos de tomate (Lycopersicon esculentum L.) y lechuga (Lactuca sativa L.). Se utilizaron contenedores de $24 \mathrm{~m}$ de longitud con 2 y $4 \%$ de pendiente y de cero a tres caídas como saltos hidráulicos de la solución nutritiva. Se midió el oxígeno disuelto en cada salto hidráulico a la entrada y salida del contenedor. En ambos cultivos se midió el peso fresco de la planta y de la raíz, peso seco y volumen de la raíz. En tomate se determinó el peso de los frutos por planta. En lechuga el máximo peso fresco de la planta se obtuvo en contenedores con $4 \%$ de pendiente y tres saltos hidráulicos. En tomate, el peso de frutos fue mayor en contenedores con $2 \%$ de pendiente y tres saltos hidráulicos, o con $4 \%$ de pendiente y dos saltos hidráulicos.

Palabras clave: Lycopersicon esculentum, Lactuca sativa, oxigenación, sistema hidropónico recirculante.

\section{SUMMARY}

The inadequate oxygenation of the nutrient solution affects the absorption of nutrients and water, and consequently affects plant growth and development. In this study, the incorporation of oxygen in a recirculating hydroponic system by two passive methods, container inclination and hydraulic jumps, was evaluated in the tomato (Lycopersicon esculentum L.) and lettuce (Lactuca sativa L.) crops. Containers of $24 \mathrm{~m}$ long were used with 2 and $4 \%$ inclination, and zero to three falls as hydraulic jumps of the nutrient solution. Dissolved oxygen in each hydraulic jump was measured at nutrient entrance and exit of the containers. Plant fresh weight, root dry weight and root volume were measured in both plant species, while fruit fresh weight only in tomato. In lettuce, the maximum plant fresh weight was obtained in containers with $4 \%$ slope and three hydraulic jumps. In tomato, fruit weight was higher in containers with $4 \%$ slope and two or three hydraulic jumps.
Index words: Lycopersicon esculentum, Lactuca sativa, root oxygenation, recirculating hydroponic system.

\section{INTRODUCCIÓN}

En el sistema hidropónico recirculante, la parte de la raíz sumergida en el líquido que fluye a lo largo del canal utiliza el oxígeno de la solución nutritiva (SN), en tanto que la parte de la raíz está expuesta a la humedad del aire lo toma directamente del ambiente (Morgan y Peckenpaugh, 2004). Un déficit de oxígeno en la solución recirculante reduce la permeabilidad de las raíces y limita la absorción de agua y nutrientes, con efectos negativos en el rendimiento de los cultivos crecidos en sistemas sin suelo (Urrestarazu y Mazuela, 2005).

En canales largos de un sistema recirculante la falta de oxígeno puede ocurrir conforme la masa radicular aumenta de tamaño, longitud y densidad (Carrasco e Izquierdo, 1996; Resh, 2006), y la misma solución actúa como barrera del intercambio gaseoso por la baja solubilidad del oxígeno, pero si la solución se mezcla con aire durante su paso en el sistema se mejora la oxigenación para las raíces (Morgan y Peckenpaugh, 2004). La pendiente longitudinal de los canales de cultivo debe variar entre 2 y $4 \%$, para que la solución nutritiva fluya adecuadamente por gravedad, y de esta manera la raíz sea eficiente en la absorción de agua y nutrientes (Carrasco e Izquierdo, 1996). Un cambio en la sección transversal de los canales produce un salto hidráulico o flujo de variación rápida que genera turbulencia en el régimen de circulación, lo que facilita la aireación de la solución y el intercambio entre el aire y el agua (Cabeza et al., 2009); así, mientras más agitada esté el agua mayor será el contenido de oxígeno disuelto (Okubo, 1980).

El objetivo de este trabajo fue evaluar la incorporación pasiva de oxígeno en la solución nutritiva, mediante cambios de flujo rápido en los contenedores de cultivo al modificar la pendiente longitudinal de los contenedores, y su efecto en variables de rendimiento de plantas de tomate (Lycopersicon esculentum L.) y lechuga (Lactuca sativa L.) crecidas en un sistema hidropónico recirculante.

\section{MATERIALES Y MÉTODOS}

La investigación se llevó a cabo en un invernadero con cubierta de polipropileno blanco con $25 \%$ de sombra, ubicado en los Valles centrales de Oaxaca ( $17^{\circ} 01^{\prime} 31^{\prime \prime} \mathrm{LN}$, $96^{\circ} 43^{\prime} 12^{\prime \prime}$ LO, y 1526 m de altitud). Los experimentos se realizaron de junio a diciembre del 2008 para el cultivo de lechuga cv. 'Satine', y de julio a diciembre del 2009 para el de tomate cv. 'Pony Express'. Las semillas de lechuga fueron germinadas en contenedores individuales de etileno vinil acetato (comercialmente conocido como "foamy") de 
$3 \times 3 \times 9 \mathrm{~cm}$. El semillero se regó inicialmente con agua y posteriormente con una la solución nutritiva de Steiner a $50 \%$ hasta que las plántulas presentaron de tres a cuatro hojas verdaderas y con una altura hasta el ápice de aproximadamente $7 \mathrm{~cm}$. El trasplante se hizo a los $35 \mathrm{~d}$, a $20 \mathrm{~cm}$ entre plantas. Las semillas de tomate fueron germinadas en contenedores de unicel con sustrato de $75 \%$ de turba Sphagnum $+25 \%$ de vermiculita. Al trasplante, que se hizo a los $35 \mathrm{~d}$ y a $60 \mathrm{~cm}$ entre plantas, el sistema radicular (raíz + sustrato) fue envuelto con esponja de poliuretano de $6 \mathrm{x}$ $15 \times 1 \mathrm{~cm}$ como medio de soporte.

En ambas especies se utilizó la técnica de la solución nutritiva recirculante (Cooper, 1978) en contenedores hechos con tubos de PVC de $7.5 \mathrm{~cm}$ de diámetro y $24 \mathrm{~m}$ de longitud. La solución nutritiva estuvo constituida con: 12.5 , 2.0, 1.7, 5.0, 1.8 y 5.0 mmoles $\mathrm{L}^{-1}$ de $\mathrm{NO}_{3}-, \mathrm{H}_{2} \mathrm{PO}_{4}^{2-}, \mathrm{SO}_{4}^{2-}$, $\mathrm{Ca}^{2+}, \mathrm{Mg}^{2+} \mathrm{y} \mathrm{K}^{+}$, respectivamente, más 1.0, 0.5, 0.3, 0.5, 0.1 y $0,05 \mathrm{mg} \mathrm{L}^{-1}$ de Fe, Mn, Zn, B, Cu, y Mo, en el mismo orden (Urrestarazu, 2004). El pH de la solución, medido en el tanque colector, se ajustó de 6.5 a 7.0 con $\mathrm{HNO}_{3}$ y la $\mathrm{CE}$ a 2.0 $\mathrm{dS} \mathrm{m}^{-1}$. La aplicación de la SN se hizo en forma intermitente con intervalos de $10 \mathrm{~min}$ de riego por $10 \mathrm{~min}$ sin riego, con gasto en el gotero de $3 \mathrm{~L} \mathrm{~h}^{-1}$, y se reemplazaba cuando el valor de la CE era menor a $1.4 \mathrm{dS} \mathrm{m}^{-1}$.

Los tratamientos fueron el resultado de la combinación de los niveles de los factores de estudio; pendientes del contenedor (2 y $4 \%$ ) y cuatro saltos hidráulicos $(0,1,2$ y 3$)$ de 5 $\mathrm{cm}$ de altura de la solución nutritiva (SN). En los dos cultivos los tratamientos se distribuyeron en un diseño completamente al azar con cuatro repeticiones. Se midió el oxígeno disuelto (\%) con un oxímetro HI 9146® (HANNA Instruments, México), en tres posiciones: junto al entorno del sistema radicular, a la entrada y a la salida del contenedor (considerado este último como salto hidráulico). El pH de la solución nutritiva se midió con un oxímetro HI 9811-5® (HANNA Instruments, México). En las plantas de tomate y lechuga se midió: peso fresco de parte aérea y de raíz, peso seco de raíz y volumen de raíz. Los pesos se registraron con una balanza Ohaus ${ }^{\circledR}$ de $2610 \mathrm{~g} \pm 0.1 \mathrm{~g}$ de capacidad. El volumen de la raíz se determinó de acuerdo con Harrington et al. (1994). Para el tomate, por ser de hábito determinado, se realizaron únicamente tres cortes de frutos y se registró su peso con la balanza antes indicada. Los datos obtenidos fueron analizados con el programa Statgraphics Plus 4 (Statistical Graphics Corp., 1999).

\section{RESULTADOS Y DISCUSIÓN}

Las pendientes del contenedor y las caídas producidas por los saltos hidráulicos mostraron diferencias significativas $(\mathrm{P} \leq 0.01)$ para el contenido de oxígeno en la solución nutritiva $(\mathrm{SN})$. En ambos cultivos, el mayor porcentaje de oxígeno en la SN se presentó en contenedores con $4 \%$ de pendiente (Cuadro 1), sobre todo cuando se combinó con tres saltos hidráulicos donde la SN de lechuga alcanzó hasta $20 \%$ más de oxígeno que en el testigo. Según Urrestarazu (2004) y Resh (2006), el contenido de oxígeno de la SN depende de la especie vegetal cultivada; por ejemplo, el pepino (Cucumis sativa L.) requiere menor concentración de oxígeno en la SN que tomate y lechuga. Esta última especie es considerada como de bajo requerimiento de oxígeno y su cultivo es ampliamente recomendado para sistemas hidropónicos recirculantes tipo NFT (Resh, 2006).

Cuadro 1. Contenido promedio de oxígeno disuelto (\%) en la solución nutritiva de tomate y lechuga, en función de la pendiente del contenedor y del número de saltos hidráulicos.

\begin{tabular}{llclcl}
\hline \multirow{2}{*}{$\begin{array}{l}\text { Número de } \\
\text { saltos de } 5 \mathrm{~cm}\end{array}$} & \multicolumn{2}{c}{ Tomate } & & \multicolumn{2}{c}{ Lechuga } \\
\cline { 2 - 3 } \cline { 5 - 6 } & $2 \%$ & $4 \%$ & & $2 \%$ & $4 \%$ \\
\hline 0 & $64.0 \mathrm{c}$ & $66.3 \mathrm{~b}$ & & $75.0 \mathrm{~b}$ & $67.1 \mathrm{c}$ \\
1 & $65.2 \mathrm{bc}$ & $71.8 \mathrm{a}$ & & $75.9 \mathrm{~b}$ & $78.1 \mathrm{~b}$ \\
2 & $68.3 \mathrm{ab}$ & $73.0 \mathrm{a}$ & & $88.0 \mathrm{a}$ & $88.6 \mathrm{a}$ \\
3 & $69.8 \mathrm{a}$ & $75.5 \mathrm{a}$ & & $90.2 \mathrm{a}$ & $95.0 \mathrm{a}$ \\
\hline
\end{tabular}

Medidas con letras iguales en la misma columna no son estadísticamente diferentes (Tukey, 0.01).

Al respecto, Carrasco e Izquierdo (1996) indican que una mayor pendiente en los contenedores incrementa la velocidad de flujo de la SN, y por ende aumenta el oxígeno disuelto en la solución como también señalaron Okubo (1980) y Cabeza et al. (2009), lo cual coincide con lo obtenido en este estudio (Cuadro 1).

Las diferencias en el contenido de oxígeno disuelto en la solución nutritiva a la entrada y salida de los contenedores no fueron significativas, diferencias que en lechuga variaron de 11 a $15 \%$ (Figura 1, A y B) y en tomate fue de $4 \%$ (Figura 1, C y D). Al respecto, Urrestarazu (2004) y Morgan y Peckenpaugh (2004) consideran que una longitud del contenedor mayor de15 $\mathrm{m}$ disminuye la oxigenación de las raíces debido a que tienen más oxígeno disponible al inicio del contenedor que al final.

En el tomate la oxigenación no tuvo efecto significativo en el crecimiento de la planta, mientras que la lechuga alcanzó su mayor peso fresco de planta (153.8 g) en contenedores con $4 \%$ de pendiente y tres saltos hidráulicos, lo que representó $180 \%$ de incremento con respecto al testigo (Cuadro 2). Un comportamiento similar se observó en el crecimiento radical de lechuga. Estos resultados muestran la importancia comercial de la oxigenación de la SN en este tipo de sistemas hidropónicos, aunque Goto et al. (1996) afirman que la aireación de la SN no mejora la producción 
A)

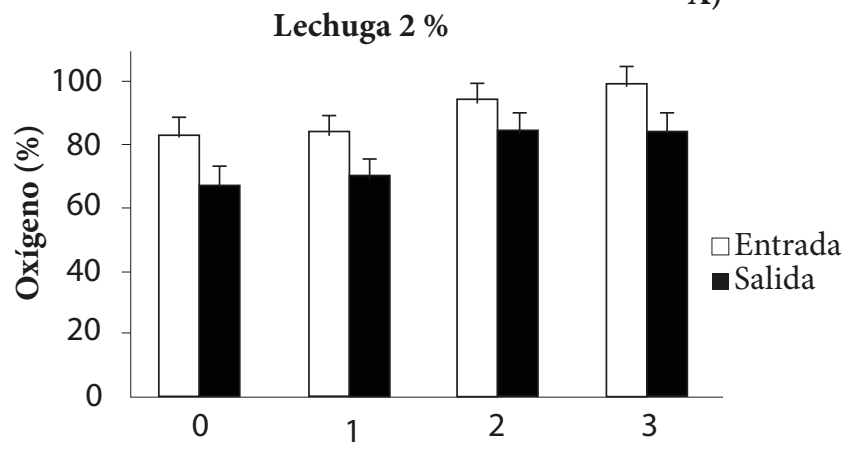

C)

Tomate $2 \%$

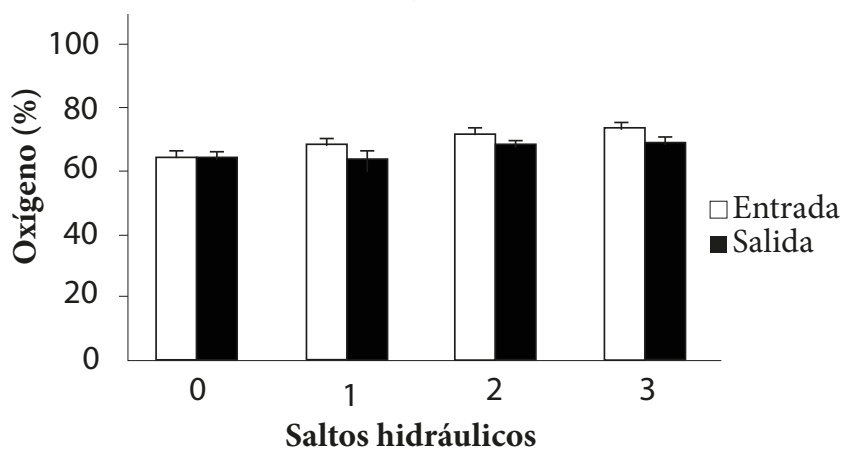

B)

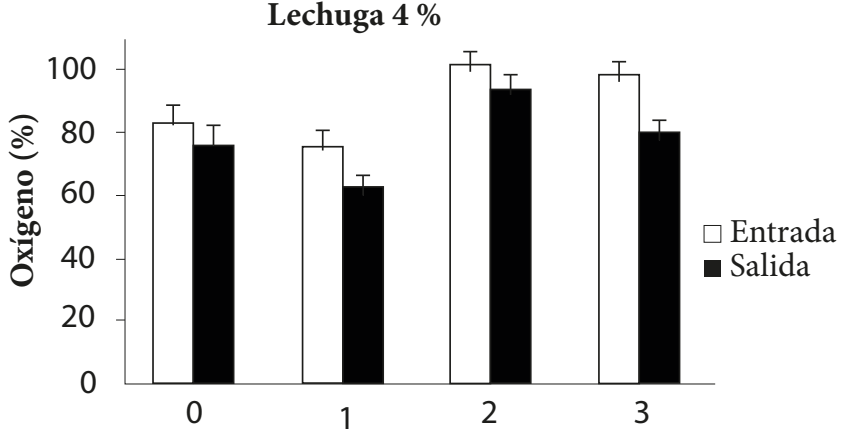

D)

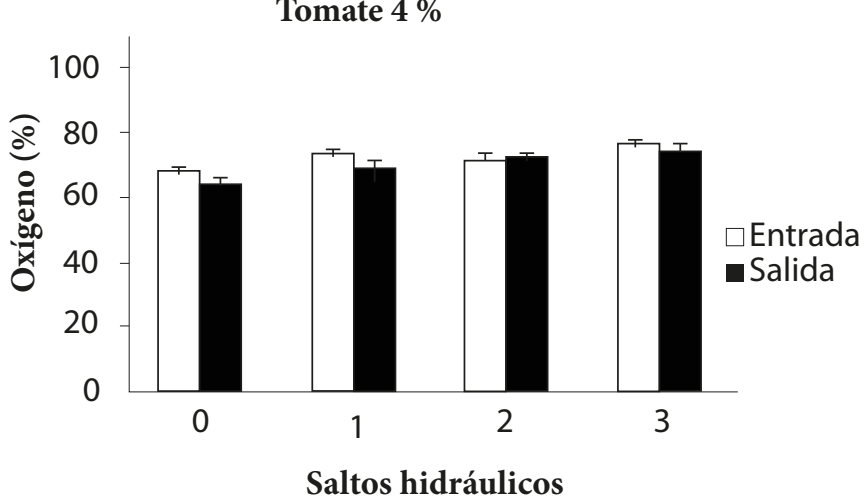

Figura 1. Contenido de oxígeno disuelto en la solución nutritiva a la entrada y salida de los contenedores de un sistema hidropónico recirculante aplicado en la producción de tomate y lechuga.

Cuadro 2. Valores promedios de variables de calidad de tomate y lechuga crecidas en sistema hidropónico recirculante, en función de la pendiente del contenedor y del número de saltos hidráulicos de $5 \mathrm{~cm}$.

\begin{tabular}{|c|c|c|c|c|c|}
\hline Pendiente & N. saltos & Peso fresco planta (g) & Peso fresco raíz (g) & Peso seco raíz (g) & Volumen raíz $\left(\mathrm{cm}^{3}\right)$ \\
\hline \multirow{4}{*}{$4 \%$} & \multirow[b]{2}{*}{0} & \multicolumn{4}{|c|}{ Tomate } \\
\hline & & $300.9 \mathrm{a}$ & $143.3 \mathrm{a}$ & $46.6 \mathrm{a}$ & $100.5 \mathrm{a}$ \\
\hline & 1 & $306.3 \mathrm{a}$ & $153.3 \mathrm{a}$ & $42.4 \mathrm{a}$ & $89.6 \mathrm{a}$ \\
\hline & 2 & $437.9 \mathrm{a}$ & $193.3 \mathrm{a}$ & $43.9 \mathrm{a}$ & $93.8 \mathrm{a}$ \\
\hline \multirow{5}{*}{$2 \%$} & 3 & $348.6 \mathrm{a}$ & $161.8 \mathrm{a}$ & $41.3 \mathrm{a}$ & $92.7 \mathrm{a}$ \\
\hline & 0 & $259.9 \mathrm{a}$ & $157.3 \mathrm{a}$ & $40.8 \mathrm{a}$ & $79.2 \mathrm{a}$ \\
\hline & 1 & $373.1 \mathrm{a}$ & $163.3 \mathrm{a}$ & $45.4 \mathrm{a}$ & $112.1 \mathrm{a}$ \\
\hline & 2 & $328.3 \mathrm{a}$ & $149.6 \mathrm{a}$ & $42.9 \mathrm{a}$ & $86.3 \mathrm{a}$ \\
\hline & 3 & $295.5 \mathrm{a}$ & $140.9 \mathrm{a}$ & $49.7 \mathrm{a}$ & $88.2 \mathrm{a}$ \\
\hline & & \multicolumn{4}{|c|}{ Lechuga } \\
\hline \multirow{4}{*}{$4 \%$} & 0 & $85.5 \mathrm{~b}$ & $36.9 \mathrm{a}$ & $0.9 \mathrm{~b}$ & $37.3 \mathrm{a}$ \\
\hline & 1 & $85.2 \mathrm{~b}$ & $36.9 \mathrm{a}$ & $1.4 \mathrm{ab}$ & $36.4 \mathrm{a}$ \\
\hline & 2 & $83.7 \mathrm{~b}$ & $36.0 \mathrm{a}$ & $1.0 \mathrm{~b}$ & $38.2 \mathrm{a}$ \\
\hline & 3 & $153.8 \mathrm{a}$ & $47.0 \mathrm{a}$ & $1.8 \mathrm{a}$ & $45.8 \mathrm{a}$ \\
\hline \multirow{4}{*}{$2 \%$} & 0 & $111.4 \mathrm{a}$ & $39.1 \mathrm{a}$ & $1.1 \mathrm{a}$ & $41.5 \mathrm{a}$ \\
\hline & 1 & $117.1 \mathrm{a}$ & $37.9 \mathrm{a}$ & $1.3 \mathrm{a}$ & $43.3 \mathrm{a}$ \\
\hline & 2 & $100.5 \mathrm{a}$ & $34.9 \mathrm{a}$ & $1.2 \mathrm{a}$ & $37.8 \mathrm{a}$ \\
\hline & 3 & $91.3 \mathrm{a}$ & $30.5 \mathrm{a}$ & $1.1 \mathrm{a}$ & $33.8 \mathrm{a}$ \\
\hline
\end{tabular}

Medias con letras iguales en la misma columna no son estadísticamente diferentes (Tukey, 0.05). 


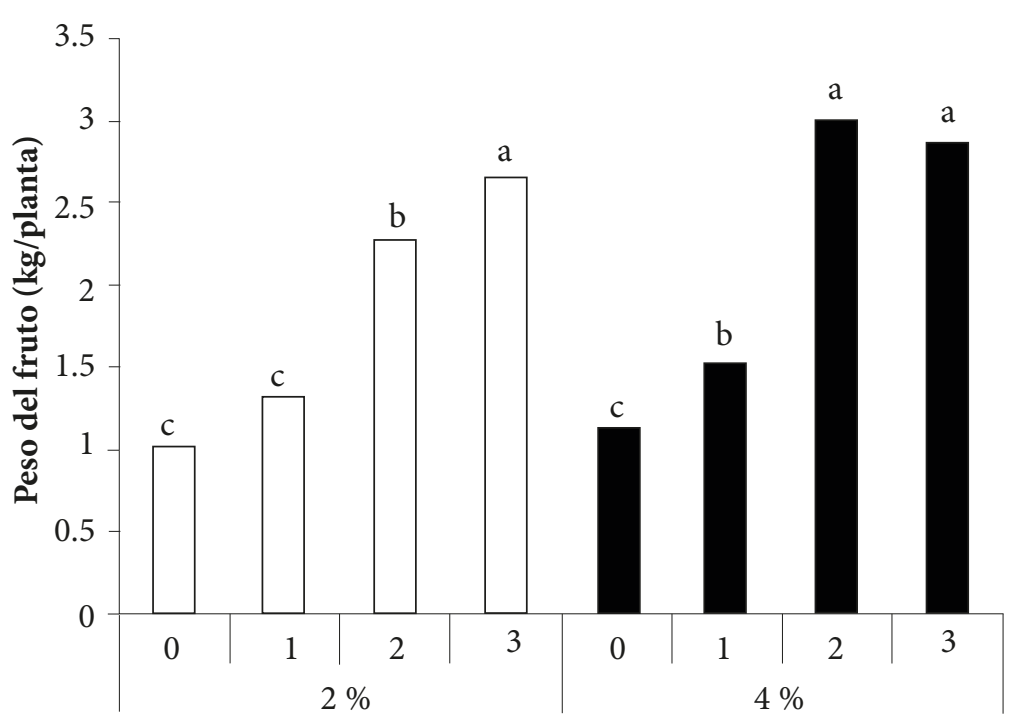

Peso del contenedor y número de saltos

Figura 2. Contenido de oxígeno disuelto en la solución nutritiva a la entrada y salida de los contenedores de un sistema hidropónico recirculante aplicado en la producción de tomate y lechuga.

de la lechuga en un sistema hidropónico flotante.

En tomate, especie en la que no hubo efecto de la oxigenación en la biomasa fresca de la planta como la observada en lechuga, se obtuvo mayor rendimiento de fruto por planta en contenedores con $2 \%$ de pendiente y tres saltos hidráulicos, o con $4 \%$ de pendiente y dos caídas (Figura 2), con aumentos superiores a $200 \%$ con respecto del testigo.

\section{CONCLUSIÓN}

Los resultados de la presente investigación muestran que el aumento de la oxigenación por métodos pasivos en sistemas hidropónicos recirculantes mediante contenedores con $4 \%$ de pendiente y dos o tres saltos hidráulicos, puede ser una opción para incrementar significativamente el rendimiento de lechuga en $180 \%$ y el de tomate en más de $200 \%$.

\section{BIBLIOGRAFÍA}

Cabeza C, J Varela, I Bove, D Freire, A C Marti, L G Sarasua, G Usera, R Montagne, M Araujo (2009) Two-layer stratified flows over pronounced obstacles at low-to-intermediate Froude numbers. Phys. Fluids 21, 044102 (2009); http://dx.doi. org/10.1063/1.3110108
Carrasco G, J Izquierdo (1996) La Técnica de la Solución Nutritiva Recirculante "NFT". Universidad de Talca, Chile. Oficina Regional de la FAO para América Latina y el Caribe. $161 \mathrm{p}$.

Cooper A (1978) Commercial Applications of NFT. Ed. Grower Books. Doughty St., London. $96 \mathrm{p}$.

Goto E, A J Both, L D Albright, R W Langhans, A R Leed (1996) Effect of dissolved oxygen concentration on lettuce growth in floating hydroponics. Acta Hort. 440:205-210.

Harrington J, J Mexal, J Fisher (1994) Volume displacement provides a quick and accurate way to quantify new root production. Tree Planters Notes 3:121-124.

Morgan L, D Peckenpaugh (2004) Hydroponic Solutions: Hydroponic Growing Tips. Vol. 1. New Moon Publishing, Co. Corvallis, OR. USA. $130 \mathrm{p}$.

Nichols M A, D J Woolley, C B Christie (2002) Effect of oxygen and carbon dioxide concentration in the root zone on the growth of vegetables. Acta Hort. 578:119-122.

Okubo A (1980) Diffusion and Ecological Problems: Mathematical Models. Lectures Notes in Biomathematics. Vol. 10. Springer Verlag, Berlin. 204 p.

Resh H M (2006) Cultivos Hidropónicos. Ed. Mundi-Prensa. 5a ed. Almería, España. 558 p.

Statistical Graphics Corp. (1999) STATGRAPHICS Plus for Windows 4.1. Statistical Graphics. Corp., Rockville, MD.

Urrestarazu M (2004) Tratado de Cultivo sin Suelo. Ed. Mundi-Prensa. Almería, España. 914 p.

Urrestarazu M, P C Mazuela (2005) Effect of slow-release oxygen supply by fertigation on horticultural crops under soilless culture. Sci. Hort. 106:484-490. 